\title{
Prenatal nicotine exposure increases connective tissue expression in foetal monkey pulmonary vessels
}

\author{
H.S. Sekhon*,\#, B.J. Proskocil*, J.A. Clark*, E.R. Spindel*
}

Prenatal nicotine exposure increases connective tissue expression in foetal monkey pulmonary vessels. H.S. Sekhon, B.J. Proskocil, J.A. Clark, E.R. Spindel. (C)ES Journals Ltd 2004.

ABSTRACT: Among the many deleterious effects of maternal smoking during pregnancy on foetal development, is a higher incidence of persistent pulmonary hypertension. The recent identification of nicotinic acetylcholine receptors (nAChR) on cells of the pulmonary vessel walls suggests that maternal smoking during pregnancy may produce morphological alterations in foetal pulmonary vasculature.

Timed-pregnant rhesus monkeys were treated with nicotine $\left(1 \mathrm{mg} \cdot \mathrm{kg}^{-1} \cdot \mathrm{day}^{-1}\right)$ delivered by subcutaneous osmotic mini-pumps from days 26-134 of gestation (term: 165 days). Lung sections from 134-day foetal monkeys were used for morphometric analysis, in situ hybridisation and immunohistochemical staining.

Following nicotine treatment, total wall and tunica adventitia thickness of airway associated vessels (AAV) increased significantly. Nicotine exposure significantly increased collagen I and III mRNA and protein in tunica adventitia in all AAV but not in tunica media. By contrast, levels of elastin protein were significantly decreased. a7 nAChR were detected in AAV fibroblasts that expressed collagen mRNA. Choline acetyltransferase, the enzyme which synthesises acetylcholine, the ligand for $\alpha 7 \mathrm{nAChR}$ was also detected in endothelium and fibroblasts.

These findings suggest that with smoking during pregnancy, nicotine is transported across the placenta and directly interacts with nicotinic acetylcholine receptors in pulmonary vessels to alter connective tissue expression and therefore produce vascular structural alterations.

Eur Respir J 2004; 23: 906-915.
*Division of Neuroscience, Oregon National Primate Research Center, Beaverton and ${ }^{\#}$ Dept of Pathology, Oregon Health \& Science University, Portland, OR, USA.

Correspondence: H.S. Sekhon, Division of Neuroscience, Oregon National Primate Research Center, 505 NW 185th Ave, Beaverton, OR 97006, USA.

Fax: 15036905384

E-mail: Sekhonh@ohsu.edu

\section{Keywords: Collagen}

nicotinic acetylcholine receptors

persistent pulmonary hypertension

pregnancy

smoking

vessels

Received: June 182003

Accepted: January 262004

This research was supported by National Institute of Health grants.
Despite the abundant evidence that maternal smoking during pregnancy adversely affects the foetus as reflected by increased incidence of preterm delivery, low birth weight and increased neonatal morbidity and mortality [1-3], 12.5\% of women continue to smoke during pregnancy [4]. In recent years, epidemiological studies have reported abnormal pulmonary function in infants $[5,6]$ who were exposed to tobacco smoke during prenatal development and that such changes persist well into adolescence [7]. These findings suggest that prenatal exposure to nicotine or cigarette smoke produces structural changes in the developing lung. This has been confirmed in animal studies in which both prenatal nicotine or cigarette smoke has been found to alter lung structure at birth [8-10]. This in turn suggests that prenatal nicotine exposure may also produce alterations in pulmonary vasculature. Consistent with this, BEARER et al. [11] have found that the incidence of persistent pulmonary hypertension was greater both in infants born to mothers who smoked during pregnancy and in infants of mothers who were exposed to passive smoke

Providing a logical basis to propose a link between prenatal nicotine exposure and alterations in pulmonary blood vessels, the current authors have previously demonstrated abundant expression of $\alpha 7$ nicotinic acetylcholine receptors $(\mathrm{nAChR})$ in foetal monkey lungs, particularly in airways, alveolar walls and blood vessels and have also reported that prenatal nicotine exposure strikingly upregulated $\alpha 7 \mathrm{nAChR}$ in fibroblasts surrounding airways and vessels [8, 12].
Given that fibroblasts surrounding pulmonary vessels and airways are the primary cells that synthesise extracellular matrix proteins and that fibroblasts express $\alpha 7 \mathrm{nAChR}$, it is likely that prenatal nicotine exposure may modify collagen expression in the developing lung. Therefore, the primary focus of the experiments reported here were to determine if maternal nicotine exposure alters morphometric dimensions of foetal pulmonary vessel walls and to begin to determine the mechanism underlying these changes. These studies will show if prenatal nicotine exposure alters pulmonary vasculature and will propose a mechanism by which, effects of prenatal nicotine exposure could persist after birth.

\section{Materials and Methods}

\section{Experimental Design}

As previously described [8], timed-pregnant monkeys were randomly assigned to nicotine-treated or control groups (three animals per group). On day 26 of gestation, Alzet 2ML4 osmotic mini-pumps (ALZA corp., Palo Alto, CA, USA) filled with nicotine tartrate (Sigma, St. Louis, MO, USA) dissolved in normal saline to deliver $1 \mathrm{mg}^{-1} \cdot \mathrm{kg}^{-1}$ body weight per day were placed subcutaneously in the midscapular region and were changed every 4 weeks. Mothers were given free access to food and water, and were weighed routinely. Foetal monkeys were delivered by cesarean section 
on day 134 of pregnancy and immediately euthanised with pentobarbital. Lungs were processed for morphometry, immunohistochemistry and in situ hybridisation as previously described [8]. Description of the effects of prenatal nicotine exposure on other aspects of these animals has been published previously [8, 12]. All animal procedures were approved by the Oregon Health and Science University Institutional Animal Care and Use Committee and conformed to all applicable regulations.

\section{Immunohistochemical staining}

Five micron sections were immunostained using primary antibodies as previously described [8, 12]. In addition, rabbit polyclonal basic fibroblast growth factor (bFGF) and rabbit transforming growth factor (TGF- $\beta 1$ ) were obtained from Santa Cruz Biotechnology (Santa Cruz, CA, USA).

\section{In situ hybridisation}

In situ hybridisation and dual immunohistochemistry/in situ hybridisation was performed as previously described [8, 12]. Generation of rhesus probes for collagen I, III and elastin were previously described [8]. For generation of complementary DNA (cDNA) probes for rhesus TGF- $\beta 1$ and bFGF, cDNAs were amplified from foetal monkey lung using the $5^{\prime}$ and 3' primers ATCGCGCCCATCTAGGTTATTTC and CGGTCGCGGGTGCTGTTGTA for TGF- $\beta 1$, and TGCCAGCATTGCCCGAGGAT and AGCAGACATTGGAAGAAAAAGTAT for bFGF. cDNA bands of the appropriate size were subcloned and sequenced. Sequences have been deposited in Genbank with respective accession numbers AF251269 and AF251270.

\section{Dual $\alpha$-bungarotoxin binding and immunohistochemistry}

The $\alpha 7$ nAChR ligand, $\alpha$-bungarotoxin $(\alpha B G T)$ was used to localise $\mathrm{nAChR}$ capable of binding ligand. Five micron sections from lung blocks fixed in Bouin's fixative were deparaffinised, and rehydrated in tris buffer saline (TBS) for $20 \mathrm{~min}$. Following incubation with binding buffer (TBS $+0.2 \%$ bovine serum albumin $+2 \%$ nonimmune horse serum) for $1 \mathrm{~h}$ at room temperature, sections were incubated overnight with the primary antibody at $4{ }^{\circ} \mathrm{C}$. After rinsing with TBS, sections were either treated with binding buffer alone or buffer containing $5 \mathrm{mM}$ nicotine at room temperature for $1 \mathrm{~h}$. Sections were incubated at room temperature with biotinylated antimouse or antirabbit antisera. After rinsing, sections were incubated with a mixture of $15 \mathrm{nM}$ Texas Red conjugated $\alpha$ BGT (Molecular Probes, Eugene, OR, USA) and avidin conjugated antimouse antibody (Vector Laboratories Inc., Burlingame, CA, USA) for $3 \mathrm{~h}$ at $37^{\circ} \mathrm{C}$.

\section{Cytokinetics}

Proliferating cell nuclear antigen (PCNA) immunostaining was used to determine proliferation index of endothelial cells and all cells in tunica media and adventitia separately. Both PCNA positive and negative cells were counted and the labelling index of each category was computed as the percent of PCNA positive cells.

\section{Morphometric measurements and image analysis}

Images were analysed as previously described [8]. For each analysis, four to five blocks per animal were used with two or more representative blocks from each lobe in the left lung. Only vessels that were cut in a cross-section were included and those with a tangential sections were not included. Data from all vessels from all animals (control or nicotine) was pooled. In cartilaginous airway associated vessels (CAAV), collagen mRNA and protein expression was discernible both in tunica adventitia and media, and measurements were made separately for each compartment and the sum of both was represented as values for the total wall. In membranous airway associated vessels (MAAV) the whole wall was included for collagen and elastin mRNA and protein expression, because in smaller airway associated vessels boundaries of tunica media and adventitia were not well demarcated. In situ hybridisation analysis was quantified as described previously and expressed either as grains per unit endothelial basement membrane or per unit area for tunica media or adventitia by counting grains and dividing by the appropriate area.

Trichrome stained images were utilised to determine vessel wall dimensions. Total wall thickness included intimal, medial, and adventitial layers. Areas of tunica media and adventitia were measured separately and corresponding length of endothelial basement membrane was also digitised. The vessel wall area was expressed per unit length of endothelial basement membrane (EMB). Haematoxylin and eosin stained sections of heart were used to measure right and left ventricle wall thickness.

\section{Statistical analysis}

Statistical analysis was performed with Number Crunching Statistical Software (Iowa, CA, USA). Values are expressed as means \pm SEM unless otherwise mentioned. Differences between the means of the treatment and control groups were determined using unpaired t-tests after testing for normal distribution equality of variance between groups. If variance between groups was not equal, then the Aspin-Welch unequal-variance t-test was used to test significance.

\section{Results}

Prenatal nicotine exposure did not significantly affect maternal body weight gain or food consumption. The effects of prenatal nicotine exposure on foetal body and organ growth in these animals has been previously reported [8, 12]. At the time of $\mathrm{C}$-section (134 days gestation) amniotic fluid nicotine levels were $15.5 \pm 3.8 \mathrm{ng} \cdot \mathrm{mL}^{-1}\left(95 \mathrm{nmol} \cdot \mathrm{L}^{-1}\right)$ which is equivalent to levels observed in heavy smokers $(>1$ pack per day) during pregnancy [13].

\section{Vessel wall dimensions and cytokinetics}

The dimensions of pulmonary vessel walls were increased following prenatal nicotine exposure. Total wall area (inclusive of tunica intima, media and adventitia) per unit EBM increased in foetal CAAV by $30 \%(\mathrm{p}<0.01)$ after nicotine treatment (fig. 1a). No significant change was seen in tunica media but the thickness of tunica adventitia increased significantly by $46 \%(\mathrm{p}<0.001)$ following nicotine exposure. The total wall area per unit EBM of MAAV increased by $28 \%$ $(p=0.005)$ and terminal bronchial associated vessels (TBV) 

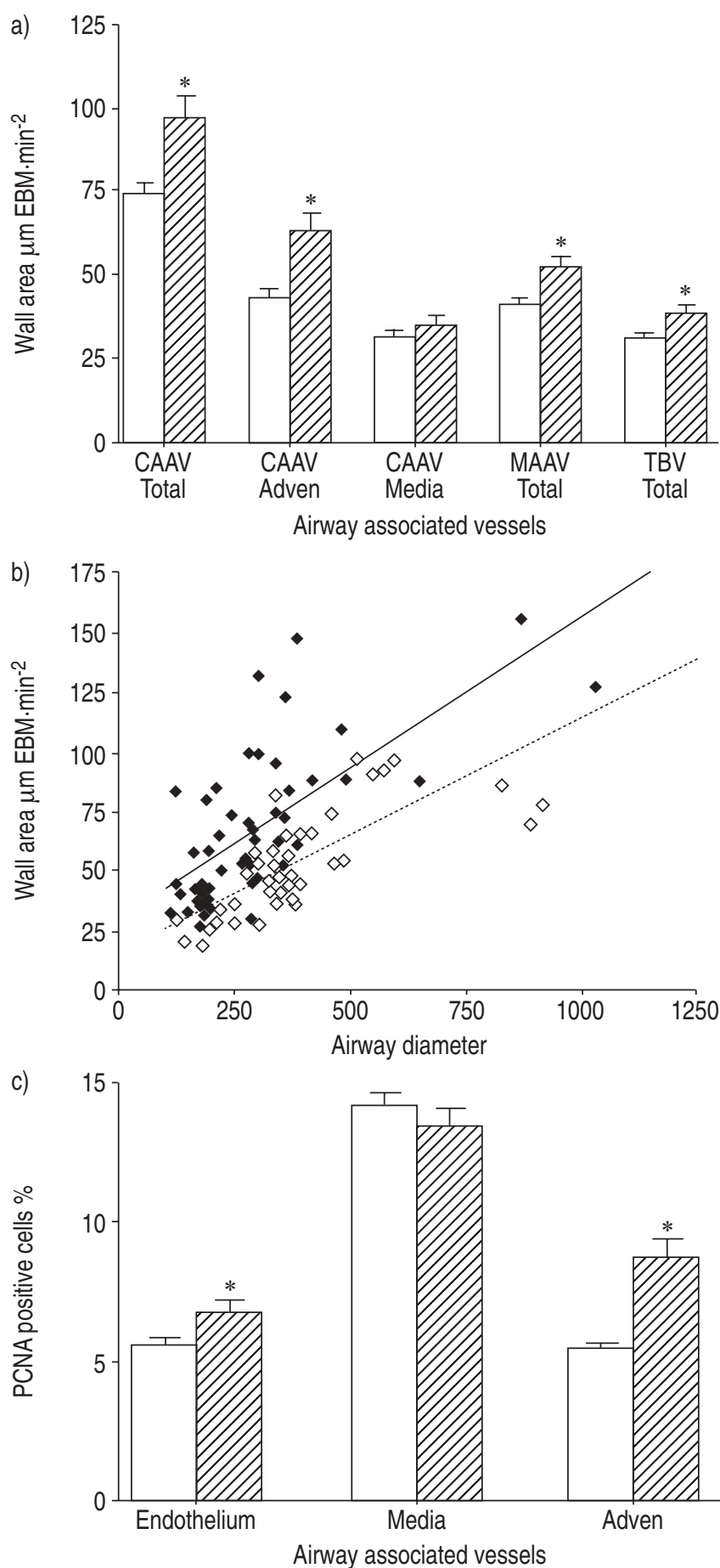

Fig. 1.-Effect of prenatal nicotine exposure on airway associated vessel size and growth. a) Effect of prenatal nicotine exposure on morphometric dimensions of vessels associated with cartilaginous airways (control sections: $\mathrm{n}=20$; nicotine-exposed: $\mathrm{n}=18$ ), membranous airways (control: $n=38$; nicotine: $n=35$ ) and terminal bronchioles (control: $\mathrm{n}=16$; nicotine: $\mathrm{n}=18$ ) associated vessels. b) Linear regression analysis showing significant $(\mathrm{p}<0.0001$ for slope $)$ increase in total airway associated vessel wall thickness versus airway diameter with nicotine exposure. c) Effect of prenatal nicotine exposure on cell proliferation in airway associated vessels. Sections randomly chosen from three nicotine treated animals $(\mathbb{Z})$ and three non-nicotine treates animals $(\square)$. EBM: endothelial basement membrane; CAAV: cartilaginous airway associated vessels; Adven: adventitia; PCNA: proliferating cell nuclear antigen; ${ }^{*}: \mathrm{p}<0.05$. increased by $24 \%$ ( $p=0.011$; fig 1a). Linear regression analysis of vessel wall thickness per unit EBM and airway diameter also showed significant differences between nicotine treated and control groups (fig. 1b). The labelling index in endothelial and adventitial layer cells was significantly increased in nicotine treated foetuses (fig. 1c). The labelling index of medial layer cells was not significantly changed. There were no significant changes in heart ventricle wall thickness (right or left) between groups (data not shown).

\section{Collagen type I expression}

In situ hybridisation showed strong collagen type I mRNA expression in cells in tunica adventitia and less in smooth muscle and endothelial cells (fig. 2a-d). Similarly, collagen protein expression assessed by immunohistochemistry was predominantly confined to the adventitial layer (fig. 2e, f). As shown in figure 2, collagen I mRNA and protein expression markedly increased in vessel walls following prenatal nicotine exposure.

Following prenatal nicotine exposure, collagen type I mRNA expression per unit EBM was significantly increased
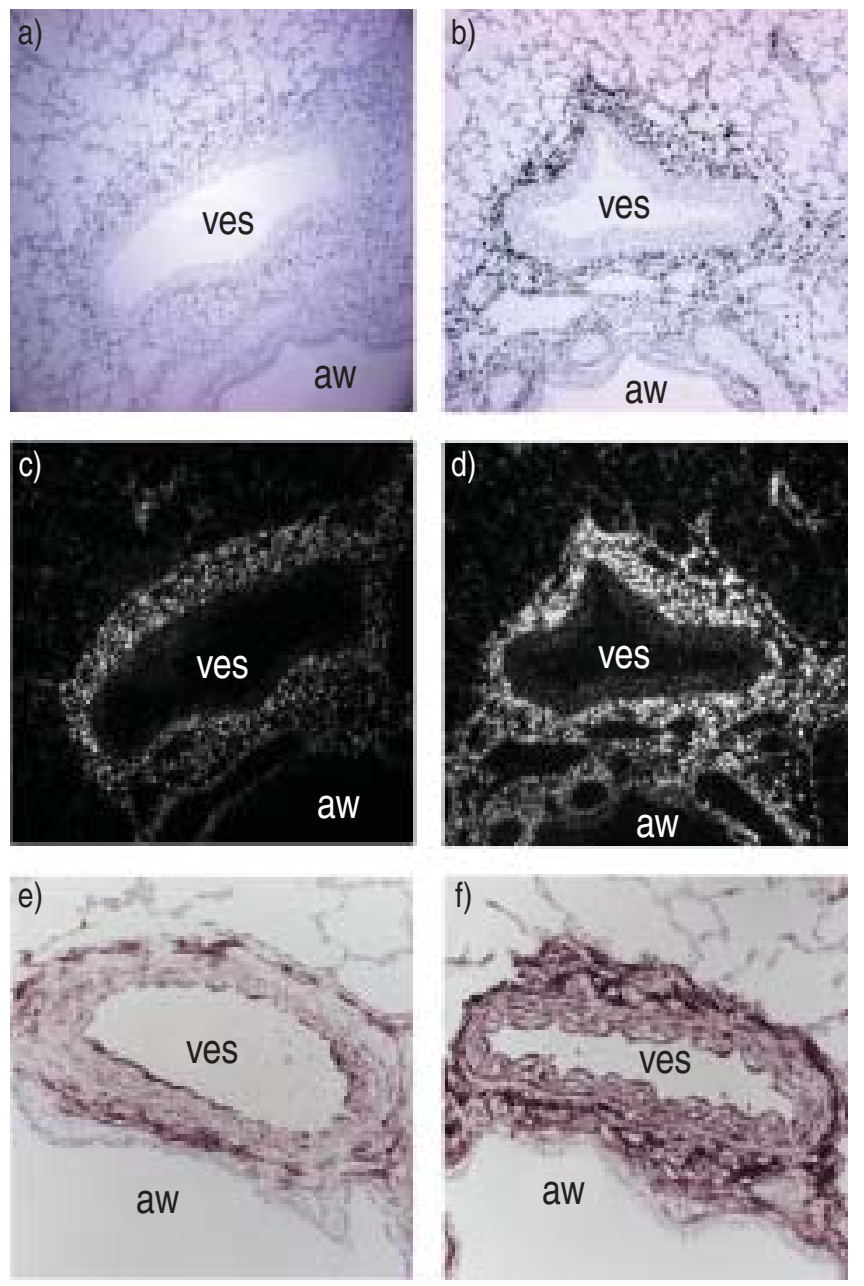

Fig. 2.-Expression of collagen type I mRNA and protein in airway associated vessels in foetal lung after prenatal nicotine exposure. a) Bright field image showing collagen $\alpha 1$ (I) mRNA expression in control lung and b) nicotine exposed lung. c) Dark field image showing collagen $\alpha 1$ (I) mRNA in control lung and d) nicotine exposed lung. Collagen type I mmunohistochemistry in e) control and f) nicotine exposed foetal lung. ves: vessel; aw: airway. 

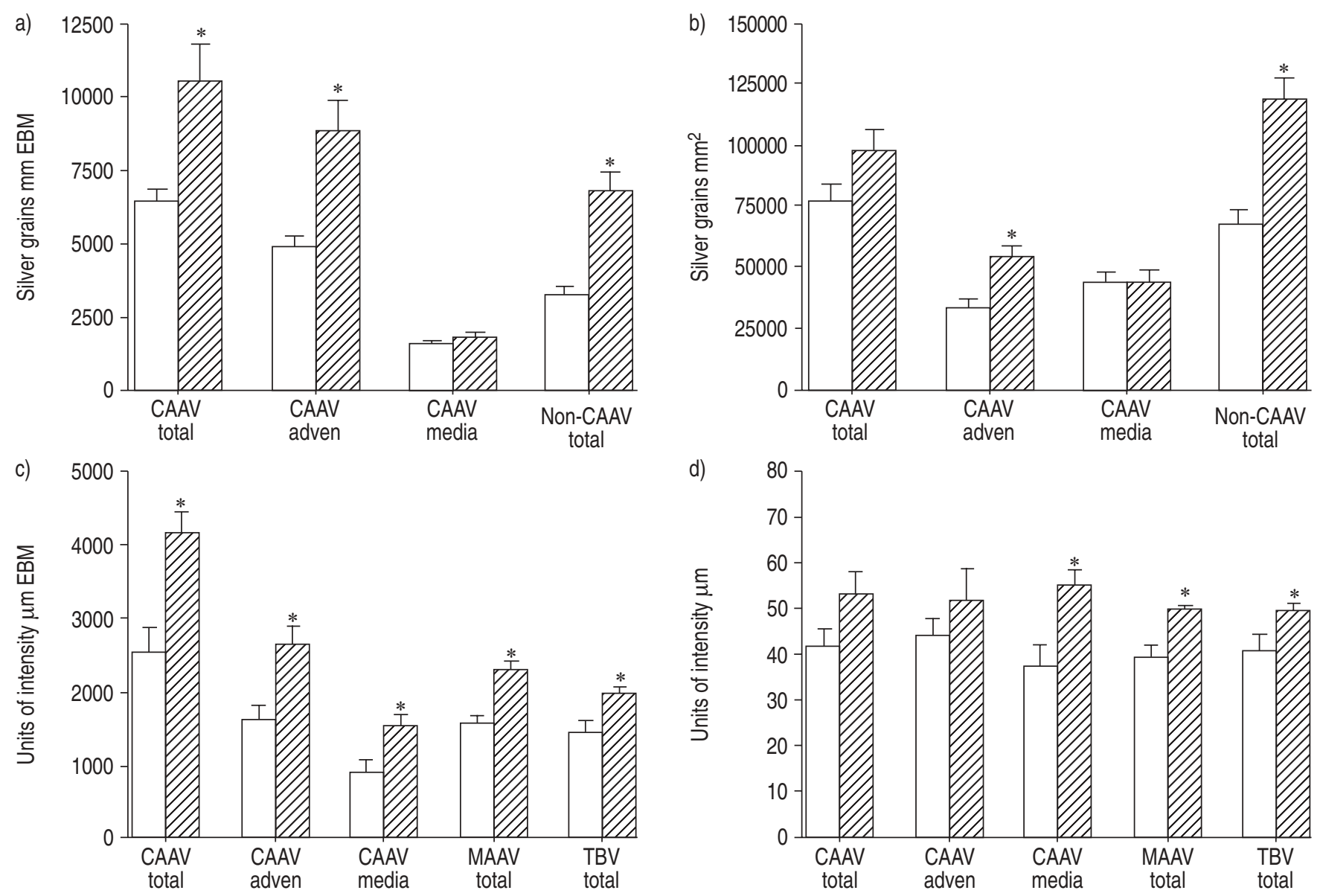

Fig. 3. - Image analysis of effects of maternal nicotine on collagen type I expression in airway associated vessels in foetal lungs. a) Quantification of collagen $\alpha 1$ (I) mRNA expression in cartilaginous airways associated vessels (CAAVs; total wall, tunica adventitia (adven) and tunica media; control sections: $n=18$; nicotine: $n=23$ ) and non-CAAVs (total wall; control: $n=32$; nicotine: $n=31$ ) airway wall per unit endothelial basement membrane (EBM). b) mRNA expression per unit vessel wall area. c) Quantification of collagen I immunostaining in CAAVs (total wall, tunica adventitia and tunica media; control: $n=11$; nicotine $n=13$ ), membranous airway associated vessels (MAAVs; total wall; control: $n=35$; nicotine: $\mathrm{n}=44$ ) and terminal bronchiole associated vessels (TBVs; control: $\mathrm{n}=21$; nicotine: $\mathrm{n}=26$ ) wall per unit EBM. d) Protein expression per unit wall area. Sections randomly chosen from three nicotine treated animals $(\mathbb{Z})$ and three non-nicotine treated animals $(\square) .{ }^{*}: \mathrm{p}<0.05$.

in vessel walls $(64 \%)$ primarily in the tunica adventitia $(80 \%)$ with no change in tunica media (fig. 3a). Collagen type I mRNA expression per unit wall area in tunica adventitia was also significantly increased (fig. 3b). In non-CAAV, collagen type I mRNA increased by $111 \%$ per unit EBM and increased by $75 \%$ per unit area in the nicotine treated group (fig. $3 a, b)$. Similar to mRNA expression, collagen type I immunohistochemical staining intensity per unit EBM was markedly increased $(66 \%)$ in CAAV wall (tunica adventitia by $64 \%$ and tunica media $71 \%$; fig. $3 \mathrm{c}$ ). Nicotine treatment also significantly increased collagen type I immunostaining intensity in MAAV and in vessels associated with terminal airways (fig. 3c, d).

\section{Collagen type III expression}

Morphologically, the distribution of collagen type III was also similar to that of collagen type I. Following prenatal nicotine exposure, collagen type III mRNA expression in the CAAV total wall per unit EBM increased by $50 \%(\mathrm{p}=0.02)$, adventitial layer per unit EBM by $67 \%$ and adventitial layer per unit wall area by $50 \%$ following nicotine treatment (fig. $4 \mathrm{a}, \mathrm{b}$ ). In non-CAAV, the number of grains in vessel wall per unit EBM $(74 \%)$ and per unit area $(49 \%)$ also increased significantly (fig. 4a, b). Immunohistochemical analysis showed a marked increase in collagen type III staining intensity in the CAAV in nicotine exposed foetal lungs. Staining intensity per unit EBM was significantly higher $(37 \%)$ in tunica adventitia (fig. 4c) but intensity per unit area remained unchanged. Compared with CAAV, a relatively greater increase was observed in MAAV and TBV walls. Similarly, in TBV, collagen III per unit EBM increased by $85 \%$ and per unit area increased by $45 \%$ after prenatal nicotine exposure (fig. $4 \mathrm{c}, \mathrm{d}$ ).

\section{Elastin}

The elastin mRNA labelling was primarily expressed in the tunica media (fig. 5a-d). Prenatal nicotine exposure increased elastin mRNA expression per unit EBM and per unit wall area in tunica media (35\% and 30\% respectively) in CAAV (figs 5, 6). Elastin mRNA expression per unit EBM in tunica adventitia was also significantly higher $(47 \%)$ in the nicotine group (fig. 6a, b). In non-CAAV (both membranous and terminal airways), silver grains per unit EBM and per unit wall area also significantly increased $(73 \%$ and $45 \%$ respectively; fig. 6a, b). 

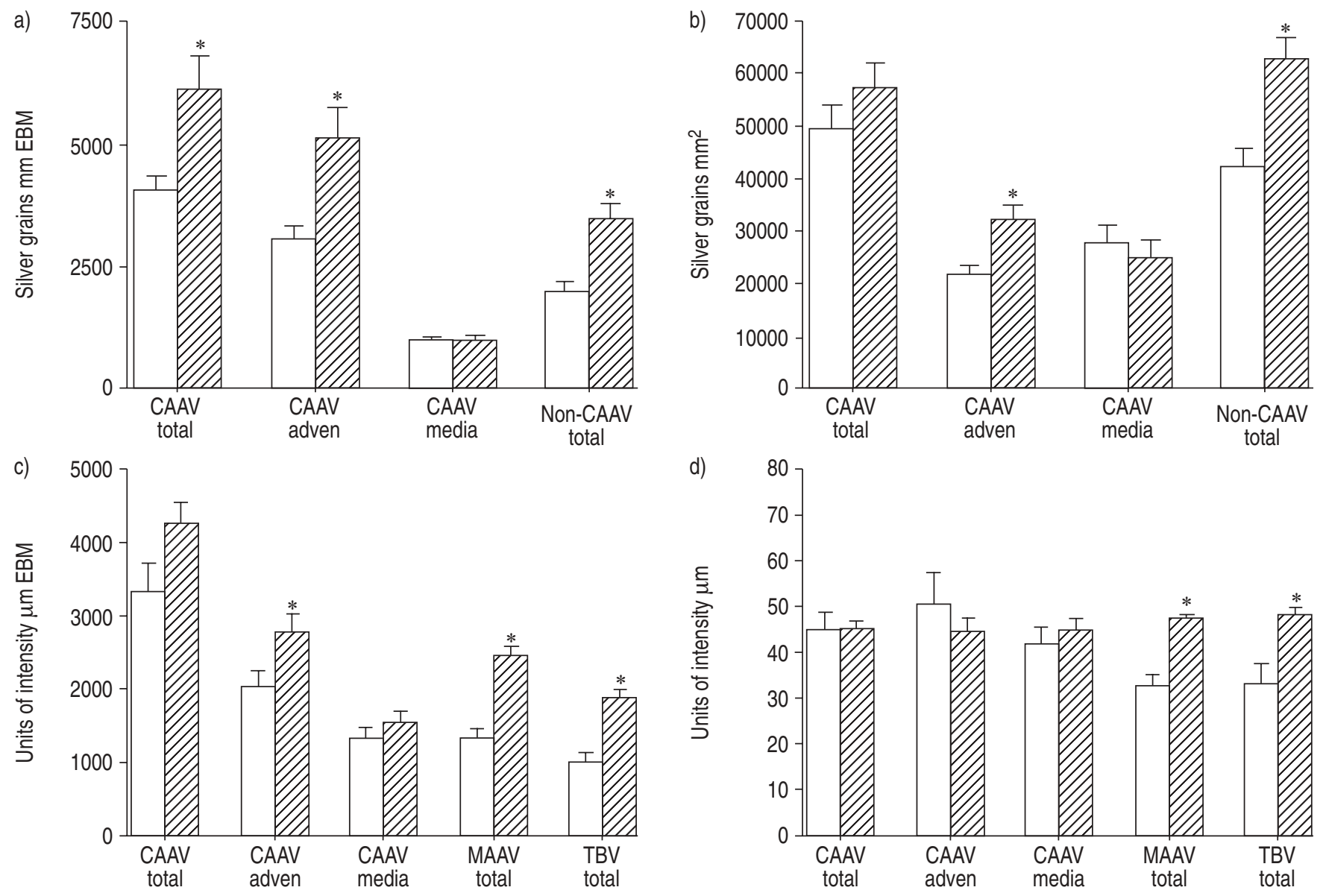

Fig. 4. - Image analysis of effects of maternal nicotine on collagen type III expression in airway associated vessels in foetal lungs. a) Quantification of collagen $\alpha 1$ (III) mRNA expression in cartilaginous airways associated vessels (CAAVs; total wall, tunica adventitia and tunica media; control sections, $n=16$; nicotine $n=22$ ) and non-CAAVs (total wall; control: $n=30$; nicotine: $n=26$ ) airway wall per unit endothelial basement membrane (EBM). b) mRNA expression per unit wall area. c) Quantification of collagen III immunostaining in CAAVs (total wall, tunica adventitia and tunica media; control: $n=20$; nicotine: $n=17$ ), membranous airway associated vessels (MAAVs; total wall; control: $n=38$; nicotine: $\mathrm{n}=35$ ) and terminal bronchiole associated vessels (TBV; control: $\mathrm{n}=16$; nicotine: $\mathrm{n}=18$ ). d) Protein expression per unit wall area. Sections were randomly chosen from three nicotine treated animals $(\mathbb{Z})$ and three non-nicotine treated animals $(\square){ }^{*}: \mathrm{p}<0.05$.

In marked contrast to the nicotine-induced increase in elastin mRNA, immunohistochemical staining (fig. 5e, f) showed a significant decrease in elastin protein deposition following nicotine treatment. Following prenatal nicotine exposure, elastin immunostaining intensity per unit wall area in tunica media decreased in CAAV $(23 \%$ and $32 \%$ respectively; fig. 6c, d). In non-CAAV, elastin protein expression was also significantly decreased (fig. 6c, d).

\section{Colocalisation of collagen and $\alpha 7$ nicotinic acetylcholine receptors expression}

If nicotine acts directly on fibroblasts to stimulate collagen expression, then $\mathrm{nAChR}$ should be present on vessel fibroblasts. As shown in figure 7, confocal microscopy using Texas-red labelled $\alpha \mathrm{BGT}$, a specific ligand for $\alpha 7 \mathrm{nAChR}$, showed strong expression of functional $\alpha 7 \mathrm{nAChR}$ in the tunica adventitia adjacent to actin-positive smooth muscle cells in the tunica media. Dual labelling with vimentin, a marker which primarily labels fibroblasts in the adventitia, suggested that the cells expressing $\alpha 7$ were most likely fibroblasts (data not shown). Trace amounts of $\alpha B G T$ binding could also be seen in the smooth muscle layer (fig. 7a). Dual immunohistochemical staining for $\alpha 7 \mathrm{nAChR}$ and in situ hybridisation for collagen mRNA similarly colocalised $\alpha 7 \mathrm{nAChR}$ expression to collagen expressing cells in the tunica adventitia (fig. 7b).

Expression of choline acetyltransferase in non-neuronal cells of blood vessels

The rich expression of $\mathrm{nAChR}$ in the adventitia raises the question of the source of acetylcholine, the ligand for $\mathrm{nAChR}$. To determine this, immunohistochemistry for ChAT, the enzyme that makes acetylcholine was performed. Dual fluorescent immunostaining for ChAT and the endothelial cell marker, factor VIII, demonstrated expression of ChAT in endothelial cells (fig. 7c). ChAT immunostaining was also present in cells in the adventitia (fig. 7d).

Tumour growth factor- $\beta 1$ and basic fibroblast growth factor $m R N A$ expression

By in situ hybridisation analysis, there was no noticeable difference in mRNA expression of TGF- $\beta 1$ and bFGF in the 

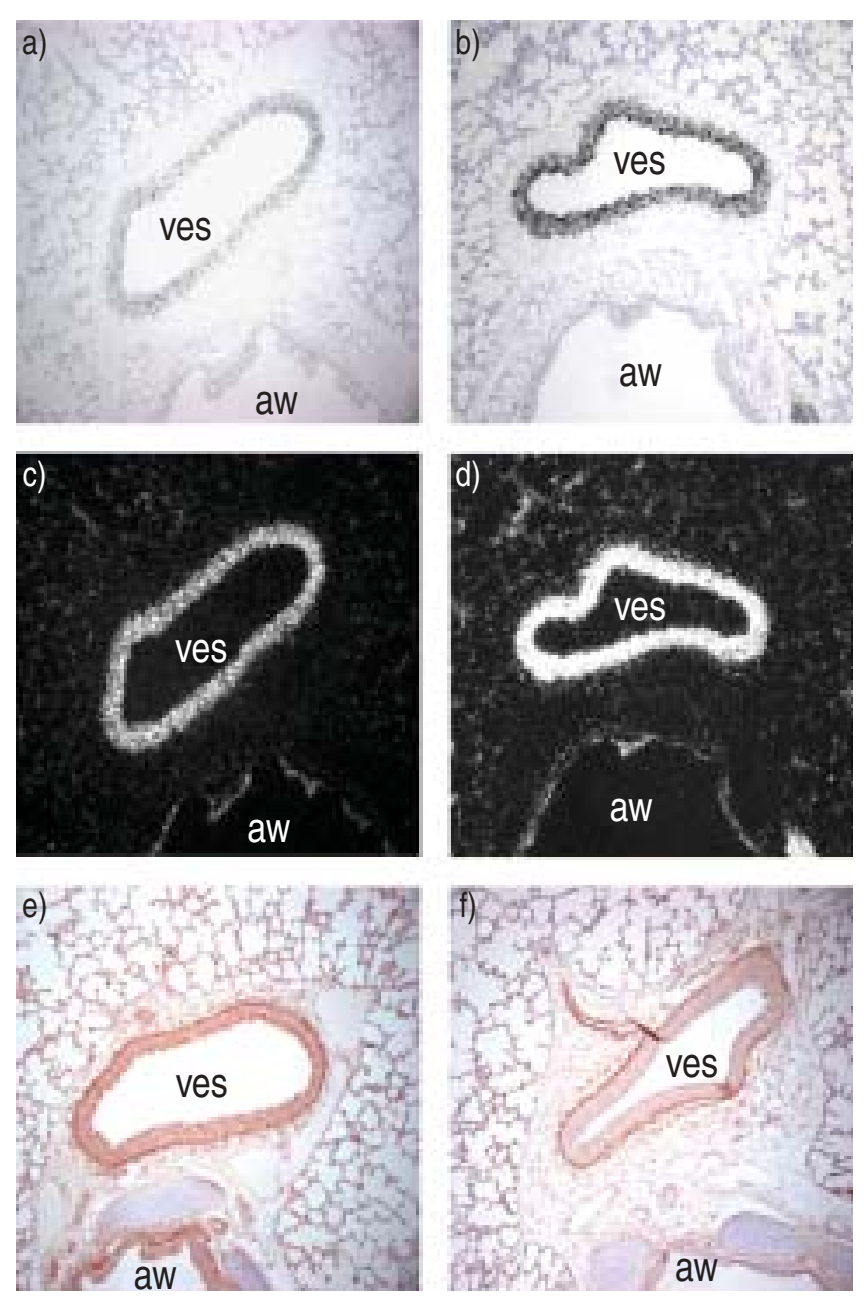

Fig. 5.-Effect of prenatal nicotine exposure on elastin expression in airway associated vessels. a) Bright field image showing elastin mRNA expression in control lung and b) nicotine exposed lung. c) Dark field image showing elastin mRNA in control lung and d) nicotine exposed lung. e) Elastin immunohistochemistry in control foetal lung and f) in nicotine exposed foetal lung. ves: vessel; aw: airway.

airway associated vessel walls between the controls and nicotine treated foetallungs (data not shown). By immunohistochemistry, TGF- $\beta 1$ protein expression appeared to be decreased or unchanged following nicotine treatment whereas bFGF appeared unchanged in the tunica adventitia (fig. 8).

\section{Discussion}

The purpose of this study was to begin to determine mechanisms by which maternal smoking during pregnancy may lead to altered pulmonary haemodynamics in infants $[11,14,15]$. In this study, the mean amniotic fluid nicotine level at the time of delivery was approximately equivalent to that observed in amniotic fluid of heavy human smokers [13]. The animals studied here were delivered at 134 days of gestation (term $=165$ days) which is $\sim 32$ weeks of human gestation a critical stage for lung development in which major vessels and airways have developed and terminal airspaces and terminal vasculature are beginning to develop.

The current authors have previously reported that prenatal nicotine exposure inhibits lung growth in foetal monkeys
[8, 12]. Despite this overall inhibition of lung growth, the authors now report that the thickness of airways associated vessels wall is significantly increased in nicotine exposed foetuses. In cartilaginous airways associated vessels, where tunica adventitia and media can be clearly delineated, there was a significant increase in thickness of the adventitia but not of tunica media. Similar observations have been reported in adults rats exposed to cigarette smoke for 2 or 3 months where the area of adventitia and intima increased in pulmonary vessels $>450 \mu \mathrm{m}$ diameter but without associated changes in the media [16]. In those rats, the volume proportion of fibroblasts increased in adventitia while smooth muscle cells in media decreased. Similarly the current authors found that the labelling index, determined by a proliferation marker was significantly increased in adventitial cells but was slightly decreased in the medial layer cells. HEESCHEN et al. [17] have similarly shown that nicotine stimulates endothelial cell proliferation. Clearly this indicates that nicotine is a key mediator of the effects of smoking on vascular connective tissue. The increases in pulmonary vessel wall thicknesses are also consistent with the findings of ELLIOT et al. [18] of increased airway wall thickness in infants dying of sudden instant death syndrome whose mothers smoked during pregnancy. The absence of measured right ventricular hypertrophy suggests unchanged pulmonary arterial pressure in the nicotine exposed group.

Prenatal nicotine exposure increased collagen I and III mRNA expression in cartilaginous and noncartilaginous airways associated vessels both per unit length of endothelium and per unit area, with largest increases in the adventitial layer following nicotine exposure. While the increase in collagen per unit length of endothelium may reflect increased vessel wall area, the increase per unit area clearly indicates increased levels of mRNA and protein. As shown in figure 7 , by immunohistochemistry, in situ hybridisation, and ligand binding techniques; fibroblasts, the predominant source of collagen in the adventitia, express abundant $\alpha 7 \mathrm{nAChR}$. Thus there is a clear path between nicotine, $\alpha 7 \mathrm{nAChR}$ on fibroblasts, and increased collagen in the adventitia. Only weak expression of $\alpha 7 \mathrm{nAChR}$ was seen in smooth muscle, consistent with the lack of effects seen in the tunica media.

In parallel with increased collagen mRNA levels, the amount of collagen type I and III protein in vessel walls also markedly increased. Collagen type I protein staining was increased both per unit endothelial basement membrane and per unit area suggesting a significant accumulation of collagen I both in tunica media and adventitia in foetuses exposed to nicotine during pregnancy. The effect of nicotine was not only limited to large vessels, the amount of collagen type I and III in vessels associated with membranous and terminal airways was also significantly increased in the nicotine treated group. MELKONIAN et al. [19] found that both main stream and side stream cigarette smoke solution altered vessel morphogenesis and increased amount of collagen type I and III and fibronectin (only side stream) in chick chorioallantoic membranes after 4 days of treatment. GiL-ACosta et al. [20] have similarly observed a significant increase in collagen I and collagen III in mouse fibroblasts treated with nicotine. Increases in adventitial collagen may also cause the vessels to be less compliant.

BEARER et al. [11] and MURASKAS et al. [15] have reported that the incidence of persistent pulmonary hypertension is greater in infants of mothers who smoked during pregnancy or who were exposed passively to cigarette smoke, suggesting that maternal smoke exposure is an important risk factor for persistent pulmonary hypertension in newborns. Whether the increases in collagen type I and III deposition observed here with nicotine similarly occurs in smoke exposed infants remains to be determined as does the question of linkage to 

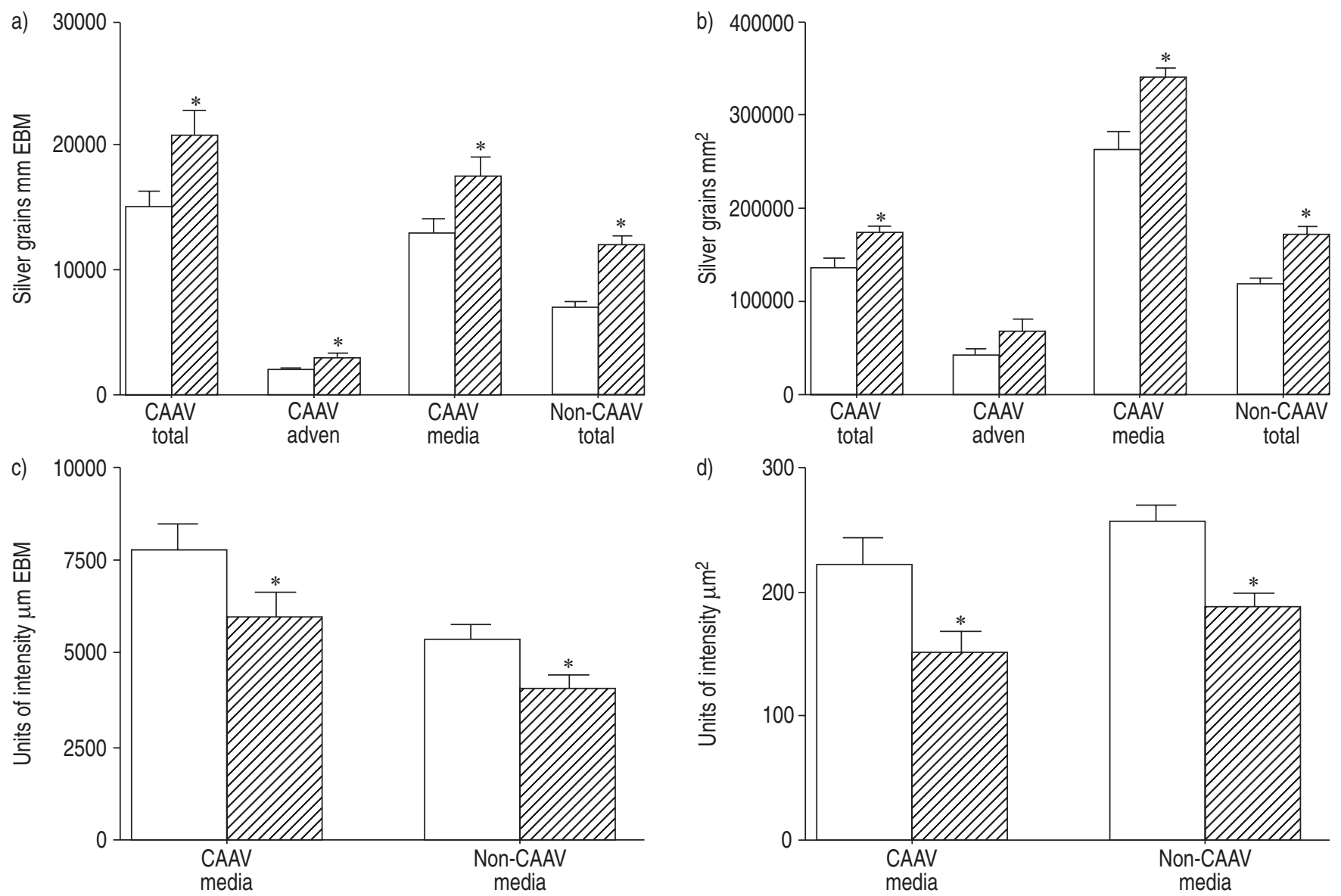

Fig. 6. - Image analysis of effects of prenatal nicotine exposure on elastin mRNA (total wall, tunica adventitia and tunica media) and protein (tunica media) expression in airway associated vessels. a) Elastin mRNA expression per unit endothelial basement membrane (EBM) in cartilaginous airway associated vessels (CAAVs; control sections: $n=26$; nicotine: $n=22$ ) and non-CAAVs (control: $n=49$; nicotine: $n=51$ ) and b) elastin mRNA expression per unit area. c) Elastin immunostaining intensity per unit EBM in CAAVs (control: $n=18$; nicotine: $n=17$ ) and nonCAAVs (control: $\mathrm{n}=23$; nicotine: $\mathrm{n}=31$ ), and d) elastin staining intensity per unit area. Sections randomly chosen from three nicotine treated animals $(\mathbb{Z})$ and three non-nicotine treated animals $(\square)$.* $^{*} \mathrm{p}<0.05$

persistent pulmonary hypertension. In that smoking during pregnancy is the leading cause of low birth weight babies, it is also possible that the alterations in collagen expression and cell number in walls caused by prenatal nicotine exposure could also be a component of why low birth weight babies may have higher incidences of cardiovascular disease as proposed by BARKER [21].

Elastin mRNA expression both per unit length of endothelium and per unit area, increased significantly but surprisingly the immunostaining intensity decreased significantly. This lack of increase in elastin protein is consistent with the lack of change in the thickness of media following nicotine exposure. The disparity between increased elastin mRNA expression and decreased elastin protein expression in pulmonary vessel walls suggests that post-transcriptional activities such as mRNA degradation, crosslinking inhibition or increased degradation may be modulated by nicotine or its metabolites. A reduction in elastic tissue fibers has also been shown in rats exposed to nicotine from day $7-21$ of postnatal life [22]. In adults, cigarette smoke is also known to increase elastase production $[23,24]$ and its activity, but whether such an increase perturbs elastin equilibrium during foetal development remains to be explored.

If nicotine is producing its effects by interacting with nicotinic receptors on collagen producing cells, what is the source of acetylcholine, the endogenous ligand for nicotinic receptors? Traditionally acetylcholine is thought to come exclusively from neurons, but while smooth muscle is richly innervated, there is little innervation of the adventitial layers surrounding blood vessels $[25,26]$. As shown in figure 7 however, there is clear expression of ChAT, the enzyme that makes acetylcholine, in both endothelial cells and in adventitial fibroblasts. This suggests that in developing lung, acetylcholine is synthesized locally by non-neuronal elements to act as a paracrine hormone. Consistent with this, WESSLER et al. [27] have described extensive expression of non-neuronal acetylcholine throughout lung, HARBERBERG et al. [28] have observed ChAT in porcine pulmonary artery endothelial cells and KAWASHIMA et al. [29] have measured acetylcholine release from cultured bovine arterial endothelial cells. This suggests that the effects of prenatal nicotine exposure on collagen expression in lung vessels, derives from the modulation of a local paracrine cholinergic signalling pathway.

Further supporting a direct effect of nicotine on fibroblasts, no changes in TGF- $\beta 1$ or bFGF were observed. This is consistent with studies showing that TGF- $\beta$ secretion following nicotine or cigarette extract treatment is either decreased or unchanged in bovine aortic endothelial cells [30], airway epithelial cells [31]. Interestingly, this is different than the results of CARTY et al. [32] who found that nicotine increased bFGF in human vascular smooth muscles cells. These cells were however harvested from post mortem cadaver arteries 

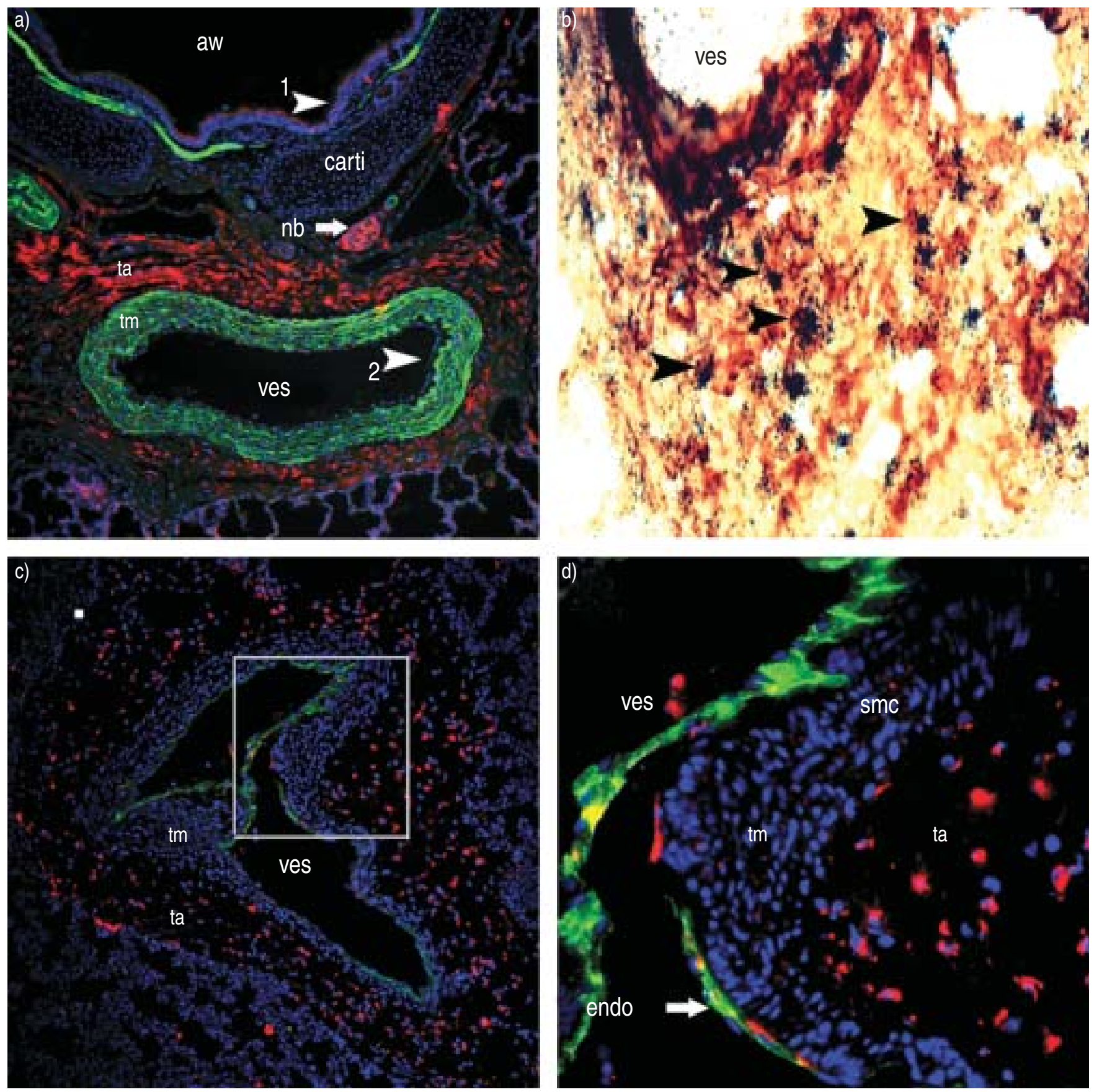

Fig. 7. - Confocal microscopy and in situ hybridisation showing expression of nicotinic receptors and choline acetyltransferase in airway vessels a) Sections were incubated with Texas-red conjugated $\alpha$-bungarotoxin ( $\alpha$-BGT: red), antismooth muscle actin (green) and DAPI nuclear stain (blue). $\alpha$-BGT binding is apparent in airway endothelium (arrowhead 1) and tunica media (tm). Smooth muscle actin is visible in airway and tunica adventitia (ta; arrowhead 2). b) Dual immunohistochemistry, in situ hybridisation using anti- $\alpha$ 7-nicotinic acetylcholine receptors (nAChR monoclonal antibody 319) and collagen type $\alpha 1$ III ${ }^{33}$ P-labelled cyclic RNA probe showing cells which express $\alpha 7$-nAChR predominantly express collagen mRNA. c) Confocal image showing expression of choline acetyltransferase (ChAT) in endothelium and adventitial layer in pulmonary vessels. Sections were incubated with anti-ChAT (red in endothelium and adventitial layers) and anti-factor VIII (green, endothelium). d) Higher power view of c) showing colocalisation of factor VIII and ChAT A (yellow). Blue: DAPI-stained nuclei; aw: airway, carti: cartilage; endo: endothelium; nb: nerve bundle; smc: smooth muscle cell layer, ves: vessel.

and thus may reflect differences between effects of nicotine in adults versus foetuses.

In summary, nicotine is readily transported across the placenta to achieve levels sufficient to interact directly with nicotinic receptors expressed on non-neuronal cells in the pulmonary vessels walls. The interaction of nicotine with nicotinic receptors on fibroblasts leads to increased collagen gene expression and alters connective tissue protein equilibrium. Such changes in regulation of connective tissue proteins pathways may lead to alterations in mechanical properties of pulmonary vessels in the growing foetus of mothers who smoke during pregnancy. These findings further support the need for active counselling and aggressive campaigns against smoking during pregnancy. 

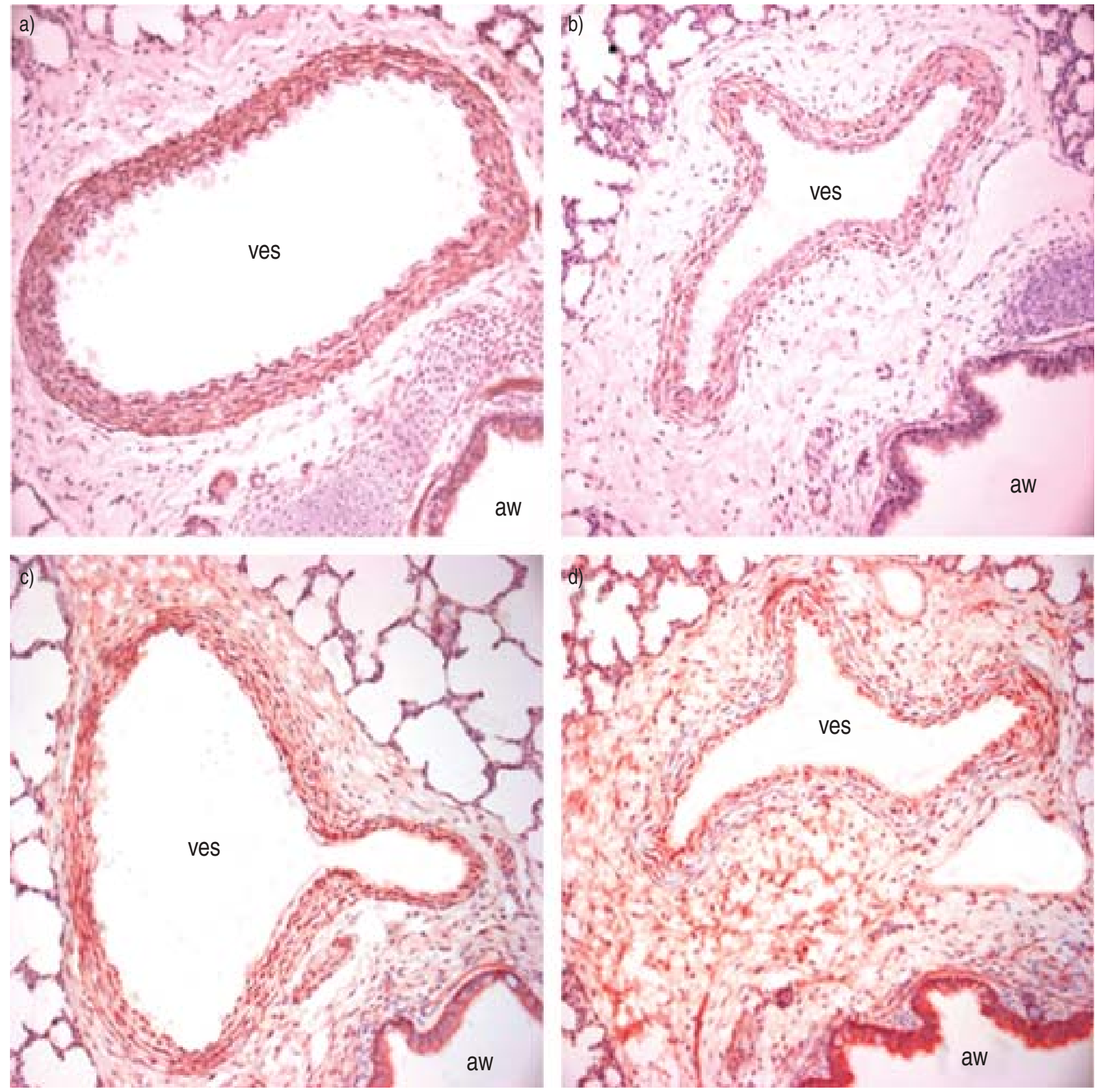

Fig. 8. - Expression of tumour growth factor (TGF)- $\beta 1$ and basic fibroblast growth factor (bFGF) in foetal monkey lung. bFGF immunostaining in a) control and b) nicotine treated foetal lung. TGF- $\beta 1$ immunostaining in (c) control and (d) nicotine-treated foetal lung. aw: airway; ves: blood vessel.

Acknowledgements. The authors would like to thank J. Fanton, D. Jacob and R. Simons of the Oregon National Primate Research Center, Division of Animal Resources for assistance with animal surgeries and timed-pregnancies. The authors would also like to thank Y. Jia of the ONPRC Molecular Biology Core for assistance in preparation of the complementary DNA probes and J. Lindstrom of the University of Pennsylvania for the generous donation of antibodies to nicotinic receptors.

\section{References}

1. Cliver SP, Goldenberg RL, Cutter GR, Hoffman HJ, Davis RO, Nelson KG. The effect of cigarette smoking on neonatal anthropometric measurements. Obstet Gynecol 1995; 85: 625-630.

2. Shah NR, Bracken MB. A systematic review and metaanalysis of prospective studies on the association between maternal cigarette smoking and preterm delivery. $\mathrm{Am}$ J Obstet Gynecol 2000; 182: 465-472.

3. Wisborg K, Kesmodel U, Henriksen TB, Olsen SF, Secher NJ. Exposure to tobacco smoke in utero and the risk of stillbirth and death in the first year of life. Am J Epidemiol 2001; 154: 322-327.

4. Mathews TJ. Smoking during pregnancy in the 1990s. Natl Vital Stat Rep 2001; 49: 1-14.

5. Tager IB, Ngo L, Hanrahan JP. Maternal smoking during pregnancy. Effects on lung function during the first 18 months of life. Am J Respir Crit Care Med 1995; 152: 977-983. 
6. Hoo AF, Henschen M, Dezateux C, Costeloe K, Stocks J. Respiratory function among preterm infants whose mothers smoked during pregnancy. Am J Respir Crit Care Med 1998; 158: 700-705.

7. Cunningham J, Dockery DW, Speizer FE. Maternal smoking during pregnancy as a predictor of lung function in children. Am J Epidemiol 1994; 139: 1139-1152.

8. Sekhon HS, Keller JA, Proskocil BJ, Martin EL, Spindel ER. Maternal nicotine exposure upregulates collagen gene expression in foetal monkey lung. Association with alpha7 nicotinic acetylcholine receptors. Am J Respir Cell Mol Biol 2002; 26: 31-41.

9. Maritz GS, Dennis H. Maternal nicotine exposure during gestation and lactation interferes with alveolar development in the neonatal lung. Reprod Fertil Dev 1998; 10: 255-261.

10. Collins MH, Moessinger AC, Kleinerman J, et al. Foetallung hypoplasia associated with maternal smoking: a morphometric analysis. Pediatr Res 1985; 19: 408-412.

11. Bearer C, Emerson RK, O'Riordan MA, Roitman E, Shackleton C. Maternal tobacco smoke exposure and persistent pulmonary hypertension of the newborn. Environ Health Perspect 1997; 105: 202-206.

12. Sekhon HS, Jia Y, Raab R, et al. Prenatal nicotine increases pulmonary alpha 7 nicotinic receptor expression and alters foetallung development in monkeys. J Clin Invest 1999; 103: 637-647.

13. Luck W, Nau H, Hansen R, Steldinger R. Extent of nicotine and cotinine transfer to the human foetus, placenta and amniotic fluid of smoking mothers. Dev Pharmacol Ther 1985; 8: 384-395.

14. O'Sullivan MJ, Kearney PJ, Crowley MJ. The influence of some perinatal variables on neonatal blood pressure. Acta Paediatr 1996; 85: 849-853.

15. Muraskas JK, Juretschke LJ, Weiss MG, Bhola M, Besinger RE. Neonatal-perinatal risk factors for the development of persistent pulmonary hypertension of the newborn in preterm newborns. Am J Perinatol 2001; 18: 87-91.

16. Liu SQ, Fung YC. Changes in the structure and mechanical properties of pulmonary arteries of rats exposed to cigarette smoke. Am Rev Respir Dis 1993; 148: 768-777.

17. Heeschen C, Jang JJ, Weis M, et al. Nicotine stimulates angiogenesis and promotes tumor growth and atherosclerosis. Nat Med 2001; 7: 833-839.

18. Elliot J, Vullermin P, Robinson P. Maternal cigarette smoking is Associated with increased inner airway wall thickness in children who die from sudden infant death syndrome. Am J Respir Crit Care Med 1998; 158: 802-806.

19. Melkonian G, Le C, Zheng W, Talbot P, Martins-Green M.
Normal patterns of angiogenesis and extracellular matrix deposition in chick chorioallantoic membranes are disrupted by mainstream and sidestream cigarette smoke. Toxicol Appl Pharmacol 2000; 163: 26-37.

20. Gil-Acosta A, Ritzenthaler JD, Roser SL, Rivera H, Aguayo SM, Roman J. Regulation of fibronectin expression in fibroblasts by nicotinic acetylcholine receptormediated signals. Am J Respir Crit Care Med 2001; 163: A546.

21. Barker DJ. Foetalorigins of cardiovascular disease. Ann Med 1999; 31: Suppl. 1, 3-6.

22. Maritz GS, Woolward K. Effect of maternal nicotine exposure on neonatal lung elastic tissue and possible consequences. S Afr Med J 1992; 81: 517-519.

23. Weitz JI, Crowley KA, Landman SL, Lipman BI, Yu J. Increased neutrophil elastase activity in cigarette smokers. Ann Intern Med 1987; 107: 680-682.

24. Gadek JE, Fells GA, Crystal RG. Cigarette smoking induces functional antiprotease deficiency in the lower respiratory tract of humans. Sci 1979; 206: 1315-1316.

25. El Bermani AW, Bloomquist EI, Montvilo JA. Distribution of pulmonary cholinergic nerves in the rabbit. Thorax 1982; 37: 703-710.

26. Canning BJ, Fischer A. Localization of cholinergic nerves in lower airways of guinea pigs using antisera to choline acetyltransferase. Am J Physiol 1997; 272: L731-L738.

27. Wessler I, Kirkpatrick CJ, Racke K. The cholinergic 'pitfall': acetylcholine, a universal cell molecule in biological systems, including humans. Clin Exp Pharmacol Physiol 1999; 26: 198-205.

28. Haberberger RV, Bodenbenner M, Kummer W. Expression of the cholinergic gene locus in pulmonary arterial endothelial cells. Histochem Cell Biol 2000; 113: 379-387.

29. Kawashima K, Watanabe N, Oohata H, et al. Synthesis and release of acetylcholine by cultured bovine arterial endothelial cells. Neurosci Lett 1990; 119: 156-158.

30. Cucina A, Corvino V, Sapienza P, et al. Nicotine regulates basic fibroblastic growth factor and transforming growth factor beta 1 production in endothelial cells. Biochem Biophys Res Commun 1999; 257: 306-312.

31. Wang H, Liu X, Umino T, et al. Cigarette smoke inhibits human bronchial epithelial cell repair processes. Am J Respir Cell Mol Biol 2001; 25: 772-779.

32. Carty CS, Soloway PD, Kayastha S, et al. Nicotine and cotinine stimulate secretion of basic fibroblast growth factor and affect expression of matrix metalloproteinases in cultured human smooth muscle cells. J Vasc Surg 1996; 24: 927-934. 